\title{
ANALYSIS OF SOLUBLE FIBRIN MONOMER AS DIAGNOSTIC MARKER FOR ACUTE MYOCARDIAL INFARCTION AND ITS CORRELATION WITH CARDIAC TROPONIN I
}

\author{
Maimun Zulhaidah Arthamin, Lydiana Parmadi, Dwi Priyadi Djatmiko, Elvin Richela Lawanto \\ Department of Clinical Pathology, Faculty of Medicine, Brawijaya University/dr. Saiful Anwar General Hospital, Malang, Indonesia. E-mail: \\ maimun70@yahoo.com
}

\begin{abstract}
The diagnosis of non-ST-Segment Elevation Myocardial Infarction (NSTEMI) is required to be early and accurate to avoid missing diagnosis and improve the rule out of Acute Myocardial Infarction (AMI) patients. There is a relationship between AMI and the state of hypercoagulation and/or thrombosis process. SFM is a prothrombotic marker found to be associated with early AMI incidence compared to CTnI, which is increased after myonecrosis. This study aimed to evaluate whether sFM can be used as a biomarker for AMI and its correlation with cTnI. A cross-sectional analytic observational study was conducted among 23 AMI patients and 27 healthy controls. Acute myocardial infarction was established using clinical, ECG, and laboratory findings. SFM levels were measured with Stago Compact Max analyzer. Statistical analysis was performed using the Spearman's correlation coefficient, ROC curve analysis, and $2 \times 2$ contingency table. A significant correlation was found between the sFM and the cTnI $(r=0.422, p<0.05)$. With an sFM cut-off level of $2.56 \mu \mathrm{g} / \mathrm{mL}$, AMI could be diagnosed with sensitivity and specificity of $82.6 \%$ and $40.7 \%$, respectively (AUC=0.638). sFM can be considered as a parameter of AMI. Similar studies with a cohort method involving a large number may be needed in the future study.
\end{abstract}

Key words: sFM, AMI, cTnI

\section{INTRODUCTION}

Acute Myocardial Infarction (AMI) occurs due to thrombus obstruction in the coronary artery. ${ }^{1}$ Plaque rupture causes subendothelial exposure, which results in tissue factor interacting with factor VII and activating factor $\mathrm{X}$, which will activate prothrombin become thrombin. ${ }^{2}$ There is a correlation between AMI, Coronary Artery Disease (CAD), and hypercoagulation state and/or thrombosis process. In this context, several prothrombotic markers are found to be associated with prediction, incidence, or prognosis of AMI such as Prothrombin Time (PT) and Activated Partial Thromboplastin (APTT) time increase significantly in AMI patients. Tissue plasminogen activator and plasminogen activator inhibitor-1 increase in AMI patients and associated with prognosis. ${ }^{3}$ Pre and postoperative fibrin monomer concentration and D-dimer increase in patients with perioperative myocardial ischemia and show strong positive correlation with postoperative $\mathrm{cTn}^{4}{ }^{4}$ Thrombus precursor proteins have been proven to be associated with prognosis in patients with Acute Coronary Syndrome (ACS) such as increased soluble fibrin in AMI patients. It has the most powerful predictor value of MI at young age ${ }^{5,6}$ Also, bedside soluble fibrin examination is useful for early identification of patients with unstable angina with non-typical electrocardiogram.

Cardiac troponin (cTn) plays an important role in the diagnosis of AMI because of its specificity for heart muscle and its sensitivity to injury. ${ }^{1}$ Diagnosis of NSTEMI is needed earlier and accurate to avoid missing diagnosis and improve rule out of AMI patients. This criterion should be fulfilled by the introduction of a new generation of cTn examination, sensitive and high sensitivity cardiac troponin (s-cTn and hs-cTn). ${ }^{8.9}$ But the introduction of hs-Tn has raised the problem of lower diagnostic specificity because this examination is positive in various nonischemic clinical conditions, including acute and chronic conditions, from the heart or not (cardiac or noncardiac). ${ }^{10}$ The need to distinguish true "thrombotic" MI from other causes of myocardial damage is a continuing challenge for appropriate treatment of the disease.

It is known that soluble fibrin monomer (sFM) is a new marker for systemic thrombus event. In the initial stage of coagulation, soluble fibrin monomers form complexes with fibrinogen and are called 
soluble Fibrin Monomer Complex (SFMC). ${ }^{7}$ Compared to other routine thromboembolic markers, sFM level has shown to be a predictor of systemic thrombus event and become a screening tool that is useful for identifying elders with an increased risk of a cardiocerebrovascular event. ${ }^{11}$ During the acute period $\mathrm{MI}$, there is a systemic imbalance between coagulability and fibrinolysis, so further studies need to be done to know the diagnostic value of sFM in AMI diagnosis. This study aimed to determine that sFM could be used as AMI biomarker and its correlation with cTnI.

\section{METHODS}

This study was conducted on the Inpatients ward of dr. Saiful Anwar General Hospital, Malang, Indonesia from March to May 2018. Sampling was done by the consecutive method. The research subjects were divided into two groups, and those were: AMI group that had been undergoing treatment at the dr. Saiful Anwar General Hospital Malang and healthy control group. Acute myocardial infarction diagnosis was made based on the latest guidelines: history, physical examination, ECG, and laboratory examination (cTnI $\geq 1)$, by the cardiologists. The inclusion criteria were male or female patients aged 18-65 years with AMI. Exclusion criteria were patients suffering from malignancy, autoimmune diseases, and sepsis. Ethical clearance was conducted by Health Research Ethical Committee Medical Faculty Brawijaya University (Number 109/EC/KPEK/04/2018).

A venous blood sample was collected in $3.2 \%$ sodium citrate and plain tube after the admission of the patient to the hospital. The blood was centrifuged at $3000 \mathrm{rpm}$ for 15 minutes to obtain the plasma and serum samples. Measurement of sFM level used plasma sample $3.2 \%$ sodium citrate with immunoturbidimetric assay STA-Liatest FM and STAGO COMPACT MAX analyzer (Diagnostica Stago,
France). The examination was based on changes in turbidity of latex microparticle suspension measured by using a photometer. Measurement of cTnI used serum sample of venous blood with the immunochromatography method. An assessment of biomarkers, including sFM and cTnI, was performed as quickly as possible after collection.

This study was an analytic observational cross-sectional. Data from this study would be analyzed statistically. Data normality test used Shapiro-Wilk after that univariate analysis was performed to determine the frequency distribution of an independent variable; those were the mean of cTnI and dependent variable, which was the mean of sFM. Data were then analyzed using SPSS computer software ver. 20. Bivariate analysis used with the Spearman test analysis technique to test the correlation between SFM and cTnI. The differential test of sFM between AMI patients and healthy controls used Mann-Whitney U Test. sFM diagnosis test used ROC curve analysis and 2x2 table with cTnI as a gold standard. P-values of 0.05 were considered statistically significant.

\section{RESULTS AND DISCUSSION}

There were 50 subjects in this study, 23 (46\%) patients with AMI, and 27 (54\%) healthy control subjects. Table 1 summarizes the characteristics of the research subjects.

Gender majority of AMI patients in this study were male $(14 ; 60.87 \%)$. There was no significant difference between the age of a patient with AMI and healthy controls. Patients diagnosed with AMI had an average age of 58.48 years. Those characteristics illustration followed sFM study on AMI that was carried out before by leko et al. ${ }^{12}$

Data normality test using Shapiro-Wilk generated $p$-value $<0.05$ in sFM and cTnI data. There was a significant correlation between sFM and CTnI ( $p<0.05 ; r=0.422)$ in AMI patients and healthy

Table 1. Characteristic differences between AMI and control groups

\begin{tabular}{llll}
\hline & AMI & Healthy control & p-value \\
\hline Sex & $\mathrm{M}=14$ & $\mathrm{M}=16$ & \\
& $\mathrm{~F}=9$ & $\mathrm{~F}=11$ & 0.094 \\
Age (Mean (SD), year) & $58.48(14.39)$ & $47.48(18.46)$ & 0.013 \\
sFM (Mean (SD) $\mathrm{\mu g} / \mathrm{mL}) \#$ & $4.13(3.38)$ & $2.91(0.95)$ & 0.000 \\
CTnI (Mean (SD) ratio)\# & $18.86(9.83)$ & $0.51(0.24)$ & \\
\hline
\end{tabular}

AMI: Acute Myocardial Infarction; sFM: Soluble Fibrin Monomer; cTnI: Cardiac Troponin I $\# p<0.05$ 
controls, and the correlation was moderate.

There was a significant difference between sFM level in AMI patients and healthy controls ( $p<0.05)$. ROC curve analysis in this study showed the ability of sFM in the diagnosis of AMI with AUC value 0.638 (Figure 1). The sensitivity and specificity of sFM with a cut-off of $2.56 \mu \mathrm{g} / \mathrm{mL}$ for AMI are 82.6 and $40.7 \%$, respectively. Low specificity in this study was caused by difficultness to get early chest pain patients who had not been accompanied by troponin increment. This was in accordance with the study of Elged et al, where in the study, patients that are included in the study were patients with acute chest pain within the first 3 hours of the attack, so that obtained the sensitivity and specificity for SFM at a cut-off value of $8.2 \mu / \mathrm{mL}$ were $87.1 \%$ and $90.0 \%$ respectively while for troponin at a cut-off value of $18 \mathrm{ng} / \mathrm{L}$ were $91.4 \%$ and $85.0 \%{ }^{7}$

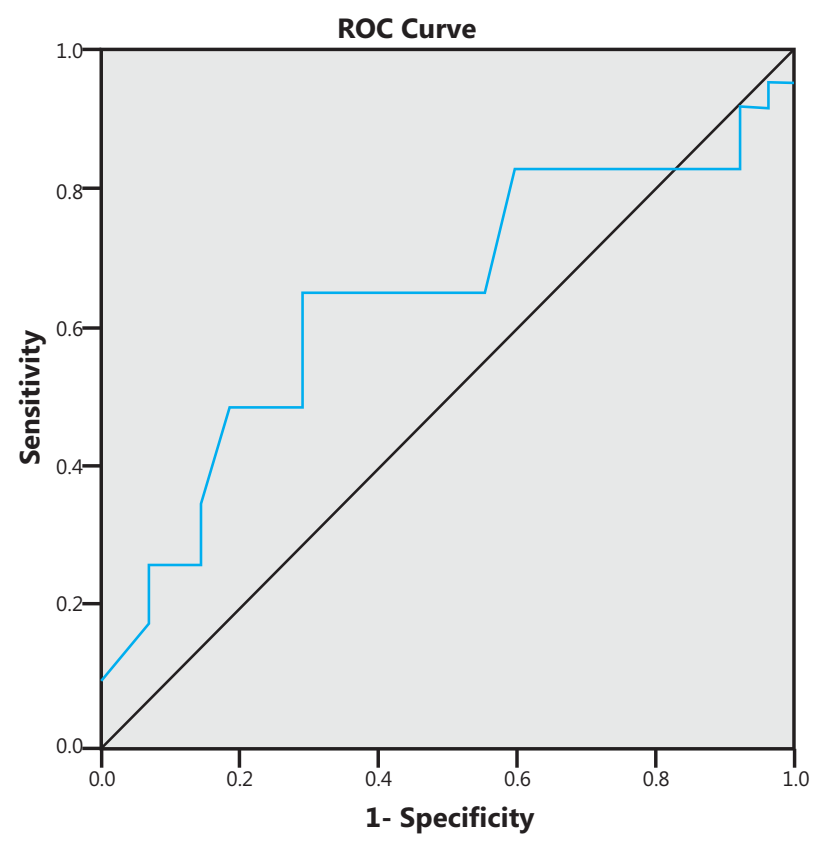

Diagonal segments are produced by ties

Figure 1. ROC curves for sFM in AMI $(A \cup C=0.638)$

Thrombus formation can occur in the hypercoagulation state. The thrombus formation is an important stage in the initial occurrence of AMI and ACS. The event starts from the conversion of prothrombin to become thrombin, which then thrombin will turn fibrinogen into fibrin later. Formation of fibrinogen derivatives, such as sFM, precedes fibrin formation in blood vessels. Other fibrin-related molecular markers are called fibrin degradation products which derived from enzymatic fission of fibrin and D-dimer. Leko et al. study showed good sFM AUC in AMI patients in the first 24 hours and was better than AUC of D-dimer, CK-MB, and Troponin, but vice versa after 24 hours. This study concluded that sFM marker was better for AMI diagnosis in the first 24 hours. ${ }^{12}$

Fibrin formation in blood vessels is preceded and followed by monomeric fibrin formation which is formed from the breakdown of fibrinogen by thrombin. ${ }^{13}$ Soluble fibrin precursors known as soluble fibrin monomer complexes or soluble fibrin appear in the blood as a result of increased in intravascular thrombin formation and reflect high risk of intravascular clot or ongoing fibrin formation. Quantitative determination of soluble fibrin monomer complex in the blood is based on immunoassays that differentiate between fibrinogen and fibrinogen derivative that is modified by thrombin. The importance of soluble fibrin monomeric diagnosis is shown in venous thromboembolism, coronary artery thrombosis, cardiopulmonary bypass, and many other conditions associated with thrombophilia and/or thrombotic condition. $^{14}$

There was a correlation between AMI and hypercoagulation state and/or thrombosis process. sFM is a prothrombotic marker that is found to be associated with the initial occurrence of AMI compared to cTnI, which will be increasing recently after the occurrence of myonecrosis. SFM is a new marker for the systemic thrombus event, both heart, and non-heart (cardiac and noncardiac). More samples were needed to further prove sFM as an early marker of thrombosis occurrence in AMI.

\section{CONCLUSION AND SUGGESTION}

sFM is considered as an alternative parameter of AMI. There was significant correlation between sFM and $c \operatorname{TnI}(p<0.05 ; r=0.422)$ in AMI patients and healthy controls. The sensitivity and specificity of sFM with the cut-off $2.56 \mu \mathrm{g} / \mathrm{mL}$ for AMI were $82.6 \%$ and $40.7 \%$, respectively (AUC $=0.638)$. Diagnosis test of sFM with cohort method and a larger number of samples is needed in future studies.

\section{Acknowledgment}

The authors acknowledge STAGO and DMC company for the supporting reagents and instrument.

\section{REFERENCES}

1. Thygesen K, Alpert JS, Jaffe AS, Simoons ML, Chaitman $B R$, White HD. Writing group on behalf of the joint 
ESC/ACCF/AHA/WHF task force for the universal definition of myocardial infarction. Third universal definition of myocardial infarction. Eur Heart J, 2012; 33: 2551-67.

2. Santos-Gallego CG, Picatoste B, Badimón JJ. Pathophysiology of acute coronary syndrome. Current atherosclerosis reports, 2014; 16(4): 401.

3. Islam S, Yakout SM, Al Daghri NM, Alhomida AS, Khan HA. Serum levels of thrombotic markers in patients with acute myocardial infarction. Int J Clin Exp Med, 2014; 7: 1059-63.

4. Bottiger BW, Snyder-Ramos SA, Lapp W, Motsch J, Aulmann M, Schweizer M, Layug EL, Martin E, Mangano DT. Association between early postoperative coagulation activation and perioperative myocardial ischaemia in patients undergoing vascular surgery. Anaesthesia, 2005; 60: 1162-67.

5. Mega JL, Morrow DA, de Lemos JA, Mohanavelu S, Cannon CP, Sabatine MS. Thrombus precursor protein and clinical outcomes in patients with acute coronary syndromes. J Am Coll Card, 2008; 51: 2422-9.

6. Saigo $M$, Waters DD, Abe $S$, Biro $S$, Minagoe $S$, Maruyama I, Tei C. Soluble fibrin, C-reactive protein, fibrinogen, factor VII, antithrombin, proteins $\mathrm{C}$ and $\mathrm{S}$, tissue factor, D-dimer, and prothrombin fragment $1+$ 2 in men with acute myocardial infarction $\leq 45$ years of age. Am J Cardiol, 2004; 94: 1410-1413.

7. Elged AA, El-Gamal RA, Bastawy S, Moselhy MS. Soluble fibrin monomer complex assay enhances early and accurate diagnosis of acute myocardial infarction. Int J Clin Exp Pathol, 2016; 9(5): 5801-9.

8. Venge $P$, Lindahl B. Cardiac troponin assay classification by both clinical and analytical performance characteristics: A study on outcome prediction. Clin Chem, 2013; 59: 976-81.

9. Apple FS, Collinson PO. IFCC task force on clinical applications of cardiac biomarkers. Analytical characteristics of high-sensitivity cardiac troponin assays. Clin Chem, 2012; 58: 54-61

10. Thygesen K, Mair J, Mueller C, Huber K, Weber M, et al. Study group on biomarkers in cardiology of the ESC working group on acute cardiac care. Recommendations for the use of cardiac troponin measurement in acute cardiac care. Eur Heart J, 2010; 31: 2197-204.

11. Tamura $H$, Hirono O, Okuyama H, Liu L, Nishiyama $S$, Takeishi $Y$, et al. Elevated serum fibrin-monomer levels are associated with long-term cerebrovascular event rates in acute ischemic stroke patients. Circ J, 2007; 71: 1573-79.

12. Ieko M, Naito S, Mika Y, Kanazawa K, Mizukami K, Sato $\mathrm{K}$, et al. Plasma soluble fibrin monomer complex as a marker of coronary thrombotic events in patients with acute myocardial infarction. Tohoku J. Exp. Med, 2009; 219:25-31.

13. Weisel JW, Litvinov RI. Mechanisms of fibrin polymerization and clinical implications. Blood, 2013; 2012.

14. Litvinov RI, Weisel JW. What is the biological and clinical relevance of fibrin. Semin Thromb Hemost, 2016: 42(4): $333-343$. 